\title{
Malignant Retroperitoneal Extra-Gastrointestinal Stromal Tumor: A Unique Entity
}

\author{
Shalini Thapar Laroia ${ }^{\mathrm{a}, \mathrm{d}}$, Taruna Yadav ${ }^{\mathrm{a}}$, Archana Rastogi ${ }^{\mathrm{b}}$, Shiv Sarin ${ }^{\mathrm{c}}$
}

\begin{abstract}
Extra-gastrointestinal stromal tumors (EGISTs) are a recently described group of tumors. A handful of less than 70 cases have been reported in English literature, so far, to the best of our knowledge. Gastrointestinal stromal tumors (GISTs) are the most common mesenchymal neoplasms of the alimentary canal. EGISTs are a unique entity, which require distinction from GISTs because, even though, they exhibit similar histology and immunohistochemistry to GISTs, they occur outside the gastrointestinal tract, i.e. in omentum, mesentery, retroperitoneum, etc. and have different behavior patterns as far as their prognosis and management are concerned. Retroperitoneal sub-group of EGISTs is extremely rare and we report such a case of primary malignant EGIST of the retroperitoneum which presented as a soft tissue mass on radiological evaluation. The tumor turned out to be a histopathological surprise, and could be distinctively labeled as EGIST only after morphological and immunohistochemical studies. It is imperative for radiologists, pathologists and oncologists, among other clinicians, to be able to recognize and understand the presentation of this group of tumors due to their rapid progression and poor prognosis, so that an early diagnosis and management may be able to improve the final disease outcome.
\end{abstract}

Keywords: Extra-gastrointestinal stromal tumor; Retroperitoneum; Computed tomography; Histopathology; Imaging findings

\section{Introduction}

A mesenchymal stromal tumor can be labeled as an extra-

Manuscript accepted for publication June 25, 2015

aDepartment of Radiology, Institute of Liver and Biliary Sciences, Sector D-1, Vasant Kunj, New Delhi 110070, India

bDepartment of Hepato-Pathology, Institute of Liver and Biliary Sciences, Sector D-1, Vasant Kunj, New Delhi 110070, India

'Department of Hepatology, Institute of Liver and Biliary Sciences, Sector D-1, Vasant Kunj, New Delhi 110070, India

${ }^{\mathrm{d} C}$ Corresponding Author: Shalini Thapar Laroia, Department of Radiology, Institute of Liver and Biliary Sciences, Sector D-1, Vasant Kunj, New Delhi 110070, India. Email: thaparshalini@gmail.com

doi: http://dx.doi.org/10.14740/wjon926w gastrointestinal stromal tumor (EGIST) by definition, when it is seen to originate outside the alimentary tract (as the name suggests) without any synchronous lesions within the gastrointestinal tract (GIT). These tumors tend to have similar characteristics to gastrointestinal stromal tumors (GISTs) on histopathology. The incidence of EGISTs is very rare and is seen to occur in less than $1 \%$ of all gastrointestinal malignancies and constitutes approximately $10 \%$ of all GIST cases [1-3]. This is probably the reason that the clinical course, imaging findings, diagnostic implications and management as well as treatment strategies for all the subtypes of EGIST are still being studied with multiple observations and no definite consensus. Of the three sub-types of EGISTs, the retroperitoneal EGIST is the rarest. A handful of less than 70 cases of retroperitoneal EGISTs have been reported in English literature, so far, to the best of our knowledge [3, 4]. In the following case study, the patient was managed with chemotherapy using imatinib mesylate, since the tumor was unresectable. However, the patient succumbed to the rapid fulminant and progressive course of this disease and died within 4 months of the initial diagnosis. We have discussed the case report of this young patient from the time of initial presentation to the management and follow-up, in order to shed some more light and analyze this particularly rare, group of primary retroperitoneal EGISTs. It is extremely challenging to diagnose these tumors without tissue biopsy. We have presented the following case in order to reiterate the importance of diagnostic capability of imaging modalities like computed tomography (CT) and magnetic resonance imaging (MRI) in evaluation of such lesions. Few studies in radiology literature have documented the presentation of retroperitoneal EGISTs and what features may be used to differentiate it, if any, from other mesenchymal tumors. We have discussed the imaging of such a mass situated in the retroperitoneum in context to its $\mathrm{CT}$ findings, along with review of imaging findings in previous similar studies.

\section{Case Report}

A 43-year-old female presented to our hospital with the complaints of progressive pain abdomen for 3 months. Abdominal pain was unrelated to food intake and was constant, dull-aching in nature. No lump was palpable on clinical examination and the abdomen was non-tender. Laboratory investigations including hematological and liver function tests were normal. 


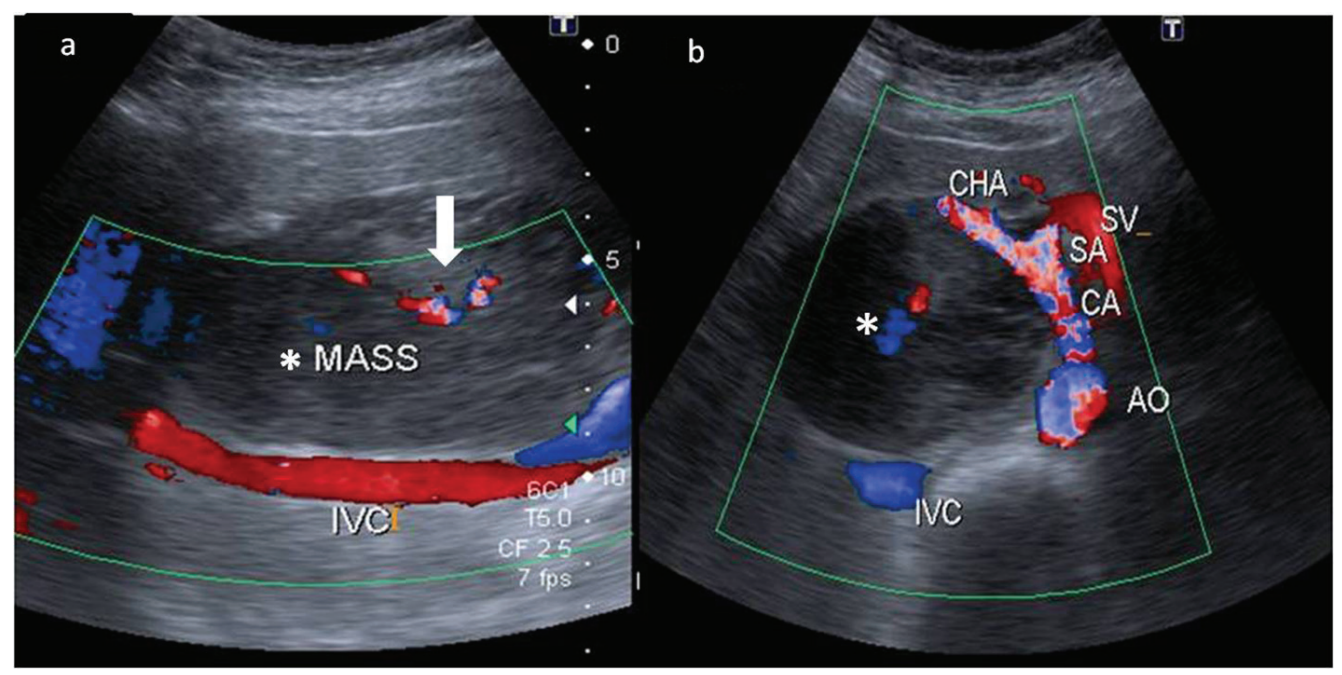

Figure 1. Color Doppler images of the mass $\left(^{*}\right)$ in the retroperitoneum. (a) Extent of the mass anterior to the inferior cava (IVC) with minimal color flow within it (white bold arrow). (b) The hypoechoic mass $\left({ }^{*}\right)$ compressing and splaying the celiac axis (CA) at its origin from the aorta (AO). The common hepatic artery (CHA) is also seen being splayed by the mass. The splenic artery (SA) and the splenic vein (SV) are in close proximity to the mass but appear spared.

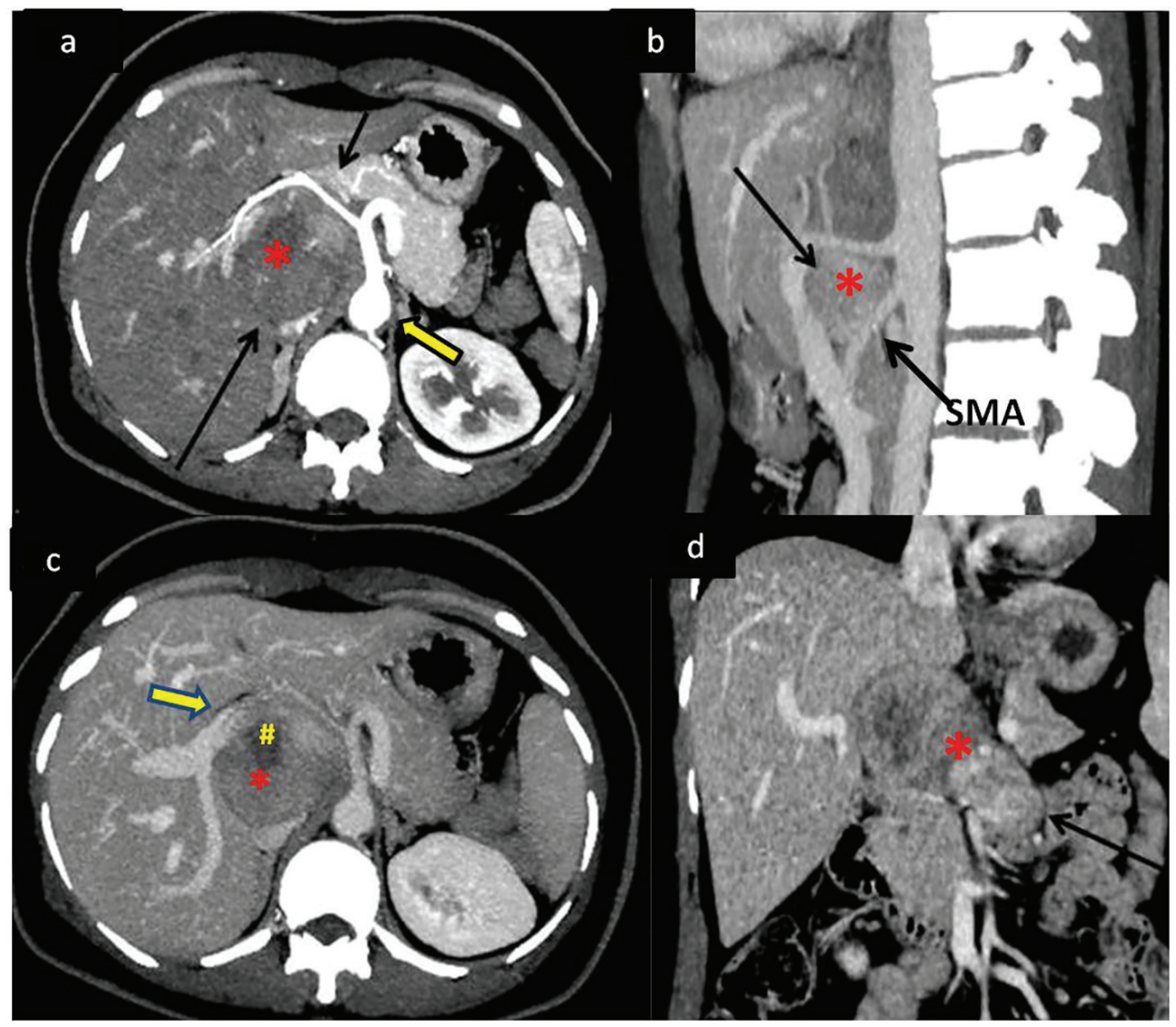

Figure 2. Dynamic contrast-enhanced CT (CECT) scan to determine extent of mass with vascular and neighboring structures involvement. (a) CECT in the arterial phase showing the soft tissue retroperitoneal mass $\left(^{*}\right)$ (bottom black arrow) anterior to the aorta (bold arrow) splaying and encasing the common hepatic artery (top black arrow). (b) Sagittal maximum intensity projection (MIP) reformatted image of the mass $\left(^{*}\right)$ splaying the celiac axis and superior mesenteric artery (SMA) at origin (arrow). (c) Portal venous phase of the dynamic CECT showing mass $\left(^{*}\right)$ displacing portal vein (bold arrow) anteriorly with areas of necrosis (\#) within it. (d) Coronal reformat showing the mass $\left({ }^{*}\right)$ extending across the midline of the abdomen (arrow). 


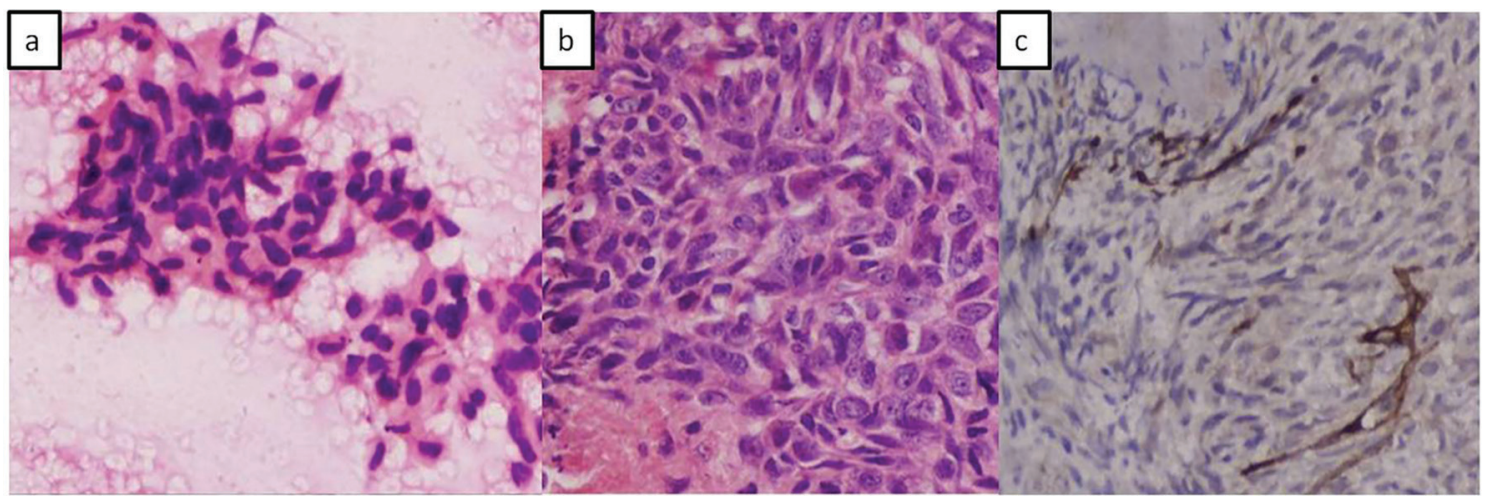

Figure 3. Photomicrographs. (a) Fine needle aspiration cytology showing fragments of tumor composed of spindle to polygonal shaped cells and elongated vesicular to hyperchromatic nuclei. (b) Cell block showing spindle cell tumor having elongated vesicular nuclei with irregular nuclear borders and prominent nucleoli. (c) Immune-histochemical staining with CD117 (kit) shows positive staining in tumor cells.

On the basis of the above physical assessment, the patient was referred for cross-sectional imaging- sonography of the abdomen, to evaluate the cause of pain. The investigation revealed a hypoechoic retroperitoneal mass sandwiched between the pancreas anteriorly and the aortocaval region posteriorly, encasing the origin of common hepatic artery (Fig. 1a, b). The patient further underwent a contrast-enhanced computed tomography (CECT) scan on a 64-slice CT scanner (Discovery$750 \mathrm{HD}$, General Electric, GE Healthcare, USA) to characterize the mass. CECT was performed by injecting $100 \mathrm{~mL}$ of intravenous contrast iomoperol (Iomeron, Bracco, Germany) using a pressure injector with bolus tracking technique and a dynamic triple phase study through an 18-gauge needle in the ante-cubital vein at a rate of $4 \mathrm{~mL} / \mathrm{s}$. In addition to a baseline non-contrast study, images were acquired during arterial phase $(20 \mathrm{~s})$, venous phase (70 s) and equilibrium phase (180 s). Thin reformatted and multiplanar images were studied before arriving at a differential diagnosis for the mass. The above CECT scan revealed a heterogeneously enhancing, lobulated solid mass lesion in the retroperitoneum with internal non-enhancing liquefied/necrotic areas anterior to the aortocaval region. The enhancement was persistent but equivalent to the phase of the dynamic study. The mass was seen to encase the celiac axis and its branches at their origin with extension along the common hepatic artery (CHA) for a small segment after origin (Fig. 2a) and along the hepatoduodenal ligament towards porta hepatis. It was seen to abut and partially encase the superior mesenteric artery (SMA) with circumferential contact of $180^{\circ}$ (Fig. 2b) as well as compress and displace the main portal vein (Fig. 2c). The mass was seen to cross the mid line towards the left side in the pre-aortic location (Fig. 2d). Mass effect on the common duct was present with resultant minimal central bilobar intrahepatic biliary radicles dilatation. Fat planes with other neighboring structures such as caudate lobe of liver, right adrenal gland and the inferior vena cava (IVC) were maintained. There was no evidence of calcification within the tumor bulk. Based on the above features of soft tissue extension and insinuation along the retroperitoneal structures and partial encasement without obvious thrombosis of the affected vessels within the abdomen, a differential diagnosis of lymphoma or retroperitoneal mesenchymal tumor was made.

Surgical management, by resection was ruled out due to the extensive involvement of vital vascular structures within the retroperitoneum by the mass. Fine needle aspiration using endoscopic ultrasound (EUS) guidance was performed for tissue diagnosis which revealed malignant spindle cells on a hemorrhagic background (Fig. 3a). Further cell block confirmed spindle cell tumor which was analyzed by immunohistochemistry (IHC) showing SMA negative and CD117 positive tumor cells. A histopathological diagnosis of malignant spindle cell tumor, likely, EGIST was made.

The patient was treated with imatinib mesylate for 3 months with regular follow-up at the hospital; however, she did not respond to therapy and succumbed to the rapid disease progression after 3 months.

\section{Discussion}

GISTs are essentially defined by their cell of origin which has been hypothesized as the interstitial cells of Cajal or related stem cell-like precursors. The cells of Cajal have been found to have role in gut motility and are found within the wall of the GIT. The gold standard diagnostic test for GIST is through IHC, which demonstrates c-kit (CD117), a tyrosine kinase growth factor receptor expressed in majority of these lesions [5-8]. GISTs present as soft tissue bulky tumors, seen as exophytic masses projecting from the wall of the gut either into the peritoneal cavity or sometimes into the lumen. The tumor extent and staging is usually identified on cross-sectional imaging by CT or MRI [9]. These neoplasms are usually sub-epithelial in location and are prone to developing complications such as fistulous communication with the bowel lumen due to invasion of the mucosal wall by tumor necrosis or mucosal ulcerations causing gastrointestinal bleed. The clinical manifestations are usually late to develop after the complications set in. Secondary spread of the disease occurs in the form of hepatic and peritoneal metastases in high grade malignant tumors and carries poor prognosis $[8,10]$.

EGIST group of tumors are a unique entity which are dis- 
Table 1. Comparison of Characteristics of Extraintestinal Gastrointestinal Tumors (EGIST and Gastrointestinal Tumor (GIST)

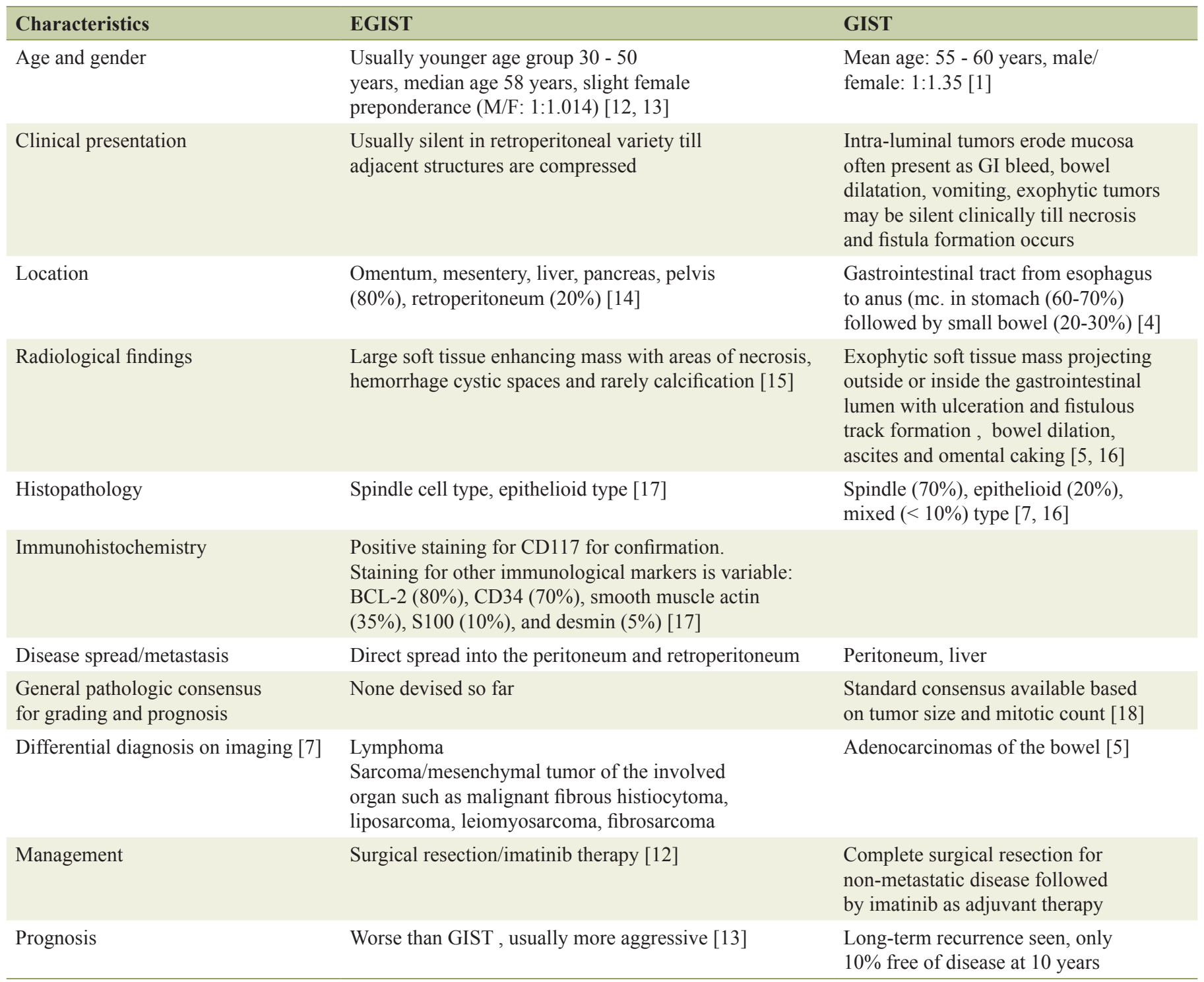

BCL-2: B-cell lymphoma 2.

tinct, yet similar to GIST in their clinical presentation, imaging, histo-pathological behavior and management strategies $[11,12]$. The term "EGIST" was coined by Reith et al in 2000 to define a unique group of stromal tumors arising outside the GIT [13]. There are many possible theories which have been suggested for the etiopathogenesis of EGIST. It has been hypothesized that, similar to GISTs, these tumors may also arise from interstitial Cajal-like cells which are common precursor cells and are also found in the retroperitoneum, omentum, pancreas and pelvis apart from the GIT. It is also believed that EGIST may have an origin from pluripotent stem cells in the above mentioned locations, outside the GIT. A distant theory also suggests that in effect, EGISTs may be an extramural extension of stromal tumors within the GIT [3]. Characteristic similarities and differences between the two entities have been listed in Table $1[1,4,5,7,12-18]$.
Till date, only 60 cases of primary EGIST of the retroperitoneum have been reported so far [3]. The objective of presenting our case of primary retroperitoneal EGIST was to highlight its clinical presentation and imaging characteristics along with the disease course showing rapid progression despite aggressive imatinib therapy. In our patient, the clinical onset of disease was insidious over a period of 3 - 4 months with increasing abdominal pain, since the mass was extraluminal in location. The pain was likely due to the neuro-vascular encasement by the mass as demonstrated in the retroperitoneum (Figs. 1a and 2a).

Extra-gastrointestinal tumors present as soft tissue complex masses with variable degrees of cystic fluid, liquefaction, necrosis and hemorrhage within the tumor bulk that would appear typically as hypoechoic or anechoic areas within an echogenic soft tissue mass on sonography and as hypoattenuating areas in- 
terspersed within an enhancing mass on contrast CT/MRI scan. Similar imaging features may be seen in lymphomas and other mesenchymal tumors in the same location; however, the differentiation from EGISTs on imaging may be made on the basis of absence of necrosis, cystic changes, hemorrhage and calcification within lymphomatous neoplasms $[19,20]$. Even though the tumor in our case did not demonstrate any calcification within it, calcification within these tumors has been described in few reports in literature $[4,6]$. Calcification is rare in untreated lymphoma of the retroperitoneum [21]. The tumor size in our patient was less than $10 \mathrm{~cm}$ in its largest dimension, which is an interesting observation compared to large malignant GISTs (average size more than $7 \mathrm{~cm}$ ) in previously demonstrated studies $[3,4,22]$. No other metastatic lesion was demonstrated on imaging. Secondary deposits in primary retroperitoneal EGISTs are rarely reported [11]. The above imaging features in a case of retroperitoneal mass lesion with internal soft tissue homogeneity and vascular encasement should prompt the radiologist to think of EGIST as a possible differential diagnosis. The endoscopic ultrasound guided fine needle aspiration cytology and cell block preparations revealed predominantly spindle cell histological pattern in our patient. The two main patterns of histology, demonstrated by retroperitoneal EGISTs, are spindle cell and epithelioid variety, showing predominantly cigar-shaped and polygonal cells, respectively $[11,12]$. The lesion demonstrated classical c-kit (CD117) positive features on IHC.

The updated consensus by ESMO, National Comprehensive Cancer Network (NCCN), and Canadian guidelines recommends CT and positron emission tomography (PET) scanning for imaging early stage lesions and the use of EUS for small incidental tumors $[5,18,23,24]$. The gold standard of diagnosis is essentially via IHC, which was also demonstrated in our study $[18,24]$. CT and EUS were used as primary modalities for staging and tissue diagnosis for our patient. Guidelines from various groups outlined above have a uniform consensus on resection of the mass, where primary GIST is more than $2 \mathrm{~cm}$ without any metastasis [25]. This is followed by further decision on the management course based on tumor size, mitotic count, and location with adjuvant use of imatinib into the management practice. As per the current worldwide practice, regarding EGIST, no such consensus has been formulated. The current most recognized mode of management of EGIST includes surgery and debulking of tumor mass wherever possible, along with the adjacent tissues which may be infiltrated by the tumor mass $[6,26]$. Tumors which are inoperable have been reportedly treated with imatinib mesylate, by some groups $[27,28]$. This drug is an inhibitor of tyrosine kinase activity of kit (CD117) protein, which in effect is a tyrosine kinase growth factor receptor expressed in more than $95 \%$ of cases of GISTs. Since the behavior pattern of EGIST is different from classical GISTs, the validity of this drug still remains non-standardized and under evaluation [29, 30]. The prognosis of EGIST appears to be less favorable than GIST [2, 13]. This appears to be due to the delayed clinical presentation leading to large tumor mass, increased proliferative indices, propensity to involve lymph nodes and distant secondaries. In our patient, few of the above prognostic factors were present such as the delayed presentation which had already led to encasement of major vasculature in the abdomen. Although the tumor size was less than $10 \mathrm{~cm}$ in diameter, our patient showed rapid progression of disease within 3 months despite aggressive imatinib therapy. These findings appear to be similar to those described by Choi et al in their paper where response and prognosis of EGIST based on tumor size have been questioned [2]. The NCCN risk table and the NIH classification system have often been used to prognosticate and manage EGISTs; however, no significant data have been demonstrated to support their appropriateness $[16,24]$.

Due to rarity of reports of primary EGISTs of retroperitoneum in literature, it is essential to study similar cases, their imaging findings and clinical progression, as discussed above, followed by their post-therapy response. It may be difficult to diagnose a retroperitoneal GIST from other mesenchymal tumors of the retroperitoneum; however, the presence of heterogeneity in the soft tissue component of a bulky mass with vascular encasement may be used as a pointer to enlist EGIST as a definite potential differential diagnosis of a solid mass in the abdominal cavity.

\section{Conclusion}

We have presented a unique case of a retroperitoneal extra-intestinal GIST, with its imaging findings and the review of literature so far with comparison of features of similar such tumors in literature. The inclusion of this group of stromal tumors is a must on the list of differential diagnosis of solid tumors arising in the abdomen and retroperitoneum and is of equal interest to clinicians, radiologists, oncologists and pathologists.

\section{Source(s) of Support}

None.

\section{Conflicts of Interest}

None.

\section{References}

1. Pidhorecky I, Cheney RT, Kraybill WG, Gibbs JF. Gastrointestinal stromal tumors: current diagnosis, biologic behavior, and management. Ann Surg Oncol. 2000;7(9):705-712.

2. Cho MY, Sohn JH, Kim JM, Kim KM, Park YS, Kim $\mathrm{WH}$, Jung JS, et al. Current trends in the epidemiological and pathological characteristics of gastrointestinal stromal tumors in Korea, 2003-2004. J Korean Med Sci. 2010;25(6):853-862.

3. Yi JH, Park BB, Kang JH, Hwang IG, Shin DB, Sym $\mathrm{SJ}$, Ahn HK, et al. Retrospective analysis of extra-gastrointestinal stromal tumors. World J Gastroenterol. 2015;21(6):1845-1850.

4. Watal P, Brahmbhatt SG, Thoriya PJ, Bahri NU. Retrop- 
eritoneal extragastrointestinal stromal tumor: radiologic pathologic correlation. J Clin Imaging Sci. 2014;4:34.

5. Levy AD, Remotti HE, Thompson WM, Sobin LH, Miettinen M. Gastrointestinal stromal tumors: radiologic features with pathologic correlation. Radiographics. 2003;23(2):283-304, 456; quiz 532.

6. Takao H, Yamahira K, Doi I, Watanabe T. Gastrointestinal stromal tumor of the retroperitoneum: CT and MR findings. Eur Radiol. 2004;14(10):1926-1929.

7. Fletcher CD, Berman JJ, Corless C, Gorstein F, Lasota J, Longley BJ, Miettinen M, et al. Diagnosis of gastrointestinal stromal tumors: A consensus approach. Hum Pathol. 2002;33(5):459-465.

8. Miettinen M, Lasota J. Gastrointestinal stromal tumors definition, clinical, histological, immunohistochemical, and molecular genetic features and differential diagnosis. Virchows Arch. 2001;438(1):1-12.

9. Gong J, Kang W, Zhu J, Xu J. CT and MR imaging of gastrointestinal stromal tumor of stomach: a pictorial review. Quant Imaging Med Surg. 2012;2(4):274-279.

10. Sandrasegaran K, Rajesh A, Rushing DA, Rydberg J, Akisik FM, Henley JD. Gastrointestinal stromal tumors: CT and MRI findings. Eur Radiol. 2005;15(7):1407-1414.

11. Abuduwayite R, Muhemaiti A, Biekemituofu H, Mohemaiti P. Malignant retroperitoneal extra-gastrointestinal stromal tumor: A case report. Br J Med Med Res. 2002;2:142-193.

12. Casella C, Villanacci V, D'Adda F, Codazzi M, Salerni B. Primary Extra-gastrointestinal Stromal Tumor of Retroperitoneum. Clin Med Insights Oncol. 2012;6:189-197.

13. Reith JD, Goldblum JR, Lyles RH, Weiss SW. Extragastrointestinal (soft tissue) stromal tumors: an analysis of 48 cases with emphasis on histologic predictors of outcome. Mod Pathol. 2000;13(5):577-585.

14. Akbulut S, Yavuz R, Otan E, Hatipoglu S. Pancreatic extragastrointestinal stromal tumor: A case report and comprehensive literature review. World J Gastrointest Surg. 2014;6(9):175-182.

15. Nishino M, Hayakawa K, Minami M, Yamamoto A, Ueda H, Takasu K. Primary retroperitoneal neoplasms: CT and MR imaging findings with anatomic and pathologic diagnostic clues. Radiographics. 2003;23(1):45-57.

16. Lech G, Korcz W, Kowalczyk E, Guzel T, Radoch M, Krasnodebski IW. Giant gastrointestinal stromal tumour of rare sarcomatoid epithelioid subtype: case study and literature review. World J Gastroenterol. 2015;21(11):33883393.

17. Suryawanshi KH, Patil TB, Damle RP, Dravid NV, Surana A. Gastrointestinal stromal tumour of small intestine presenting as a mesenteric mass. J Clin Diagn Res. 2014;8(6):FD14-16.

18. Blackstein ME, Blay JY, Corless C, Driman DK, Riddell R, Soulieres D, Swallow CJ, et al. Gastrointestinal stromal tumours: consensus statement on diagnosis and treatment. Can J Gastroenterol. 2006;20(3):157-163.
19. Carroll BA, Ta HN. The ultrasonic appearance of extranodal abdominal lymphoma. Radiology. 1980;136(2):419425.

20. Soyer P, Van Beers B, Teillet-Thiebaud F, Grandin C, Kazerouni F, Barge J, Pringot J, et al. Hodgkin's and nonHodgkin's hepatic lymphoma: sonographic findings. Abdom Imaging. 1993;18(4):339-343.

21. Yeung KW, Liu GC, Sheu RS, Kuo YT, Yang CW. Calcification in retroperitoneal non-Hodgkin lymphoma prior to treatment: a case report. Gaoxiong Yi Xue Ke Xue Za Zhi. 1995;11(5):290-294.

22. Ghanem N, Altehoefer C, Furtwangler A, Winterer J, Schafer O, Springer O, Kotter E, et al. Computed tomography in gastrointestinal stromal tumors. Eur Radiol. 2003;13(7):1669-1678.

23. Cao H, Zhang Y, Wang M, Shen DP, Sheng ZY, Ni XZ, $\mathrm{Wu} Z \mathrm{Y}$, et al. Prognostic analysis of patients with gastrointestinal stromal tumors: a single unit experience with surgical treatment of primary disease. Chin Med J (Engl). 2010;123(2):131-136.

24. Demetri GD, Benjamin RS, Blanke CD, Blay JY, Casali P, Choi H, Corless CL, et al. NCCN Task Force report: management of patients with gastrointestinal stromal tumor (GIST) - update of the NCCN clinical practice guidelines. J Natl Compr Canc Netw. 2007;5(Suppl 2):S1-29; quiz S30.

25. Casali PG, Blay JY. Gastrointestinal stromal tumours: ESMO Clinical Practice Guidelines for diagnosis, treatment and follow-up. Ann Oncol. 2010;21(Suppl 5):v98102.

26. Blanke CD, Demetri GD, von Mehren M, Heinrich MC, Eisenberg B, Fletcher JA, Corless CL, et al. Long-term results from a randomized phase II trial of standard- versus higher-dose imatinib mesylate for patients with unresectable or metastatic gastrointestinal stromal tumors expressing KIT. J Clin Oncol. 2008;26(4):620-625.

27. Dematteo RP, Ballman KV, Antonescu CR, Maki RG, Pisters PW, Demetri GD, Blackstein ME, et al. Adjuvant imatinib mesylate after resection of localised, primary gastrointestinal stromal tumour: a randomised, double-blind, placebo-controlled trial. Lancet. 2009;373(9669):10971104.

28. Du CY, Shi YQ, Zhou Y, Fu H, Zhao G. The analysis of status and clinical implication of KIT and PDGFRA mutations in gastrointestinal stromal tumor (GIST). J Surg Oncol. 2008;98(3):175-178.

29. Li J, Gong JF, Wu AW, Shen L. Post-operative imatinib in patients with intermediate or high risk gastrointestinal stromal tumor. Eur J Surg Oncol. 2011;37(4):319-324.

30. Jang SH, Kwon JE, Kim JH, Lee JY, Kim SG, Kim JS, Jung HC, et al. Prediction of Tumor Recurrence in Patients with Non-Gastric Gastrointestinal Stromal Tumors Following Resection according to the Modified National Institutes of Health Criteria. Intest Res. 2014;12(3):229235. 